\title{
DORMIR, MANGER ET PARLER : LIEN SYMBOLIQUE
}

\section{ARTICLE ORIGINAL}

PALLADINO, Ruth Ramalho Ruivo1, SOUZA, Luiz Augusto de Paula², PALLOTTA, Mara Lucia ${ }^{3}$, COSTA, Rogério da ${ }^{4}$, CUNHA, Maria Claudia ${ }^{5}$

PALLADINO, Ruth Ramalho Ruivo. Et al. Dormir, manger et parler : lien symbolique. Revista Científica Multidisciplinar Núcleo do Conhecimento. An 06, Ed. 08, vol. 06, p. 153 à 170. Août 2021. ISSN: 2448-0959, Lien d'accès: https://www.nucleodoconhecimento.com.br/psychologie-fr/lien-symbolique, $\quad$ DOI: 10.32749/nucleodoconhecimento.com.br/psychologie-fr/lien-symbolique

\section{RÉSUMÉ}

Le sommeil, la nourriture et le langage sont des piliers de la vie saine des enfants, sont étroitement liés dès la naissance et constituent la structure dynamique du développement de l'enfant. Ce sont les effets de conditions interdépendantes: organiques, psychiques et sociales, qui impliquent l'enfant et résultent, simultanément, d'héritages organiques et symboliques. Ce dernier surdétermine et module l'interaction de l'enfant avec l'environnement, en particulier avec l'autre humain qui s'y trouve. Cet héritage dessinera des modèles de conduite et de comportement qui peuvent souvent contribuer à des changements qui

\footnotetext{
${ }^{1}$ Doctorat en psychologie clinique, professeur de doctorat du programme d'études supérieures en communication et santé humaines, Faculté des sciences humaines et de la santé, PUC-SP (ORCID: https://orcid.org/0000-0001-8466-838X).

2 Doctorat en psychologie clinique, professeur titulaire du programme d'études supérieures en communication humaine et santé, Faculté des sciences humaines et de la santé, PUC-SP (ORCID: https://orcid.org/0000-00034968-9753).

${ }^{3}$ Doctorant en Communication Humaine et Santé à PUC-SP (ORCID : https://orcid.org/0000-0001-5986-9657).

${ }^{4}$ Doctorat en philosophie, ph.d. professeur du Programme d'études supérieures en communication et sémiotique, PUC-SP (ORCID: https://orcid.org/0000-0002-6807-4263).

${ }^{5}$ Doctorat en psychologie clinique, professeur titulaire du programme d'études supérieures en communication et santé humaines, Faculté des sciences humaines et de la santé, PUC-SP (ORCID: https://orcid.org/0000-00033198-6995).
}

$\mathrm{RC}: 98235$

Disponible en: https://www.nucleodoconhecimento.com.br/psychologie-fr/liensymbolique 
compromettent, dans une certaine mesure, le développement global de l'enfant. Dans la clinique pour enfants, la description des troubles du développement, du plus léger au plus grave, comprend, en règle générale, des aspects liés à la nourriture, au sommeil et au langage, ce qui suggère alors une triade de base, interrogeant les cliniciens sur la possibilité qu'il y ait, plus qu'une simple coïncidence, une corrélation entre les fonctions biologiques fondamentales. Si tel est le cas, il sera important que le clinicien s'approprie cette perspective, car l'implication déterminera probablement les particularités des procédures de diagnostic et de traitement. Dans ce sens, il convient d'approfondir et de discuter du développement de ces fonctions (sommeil, alimentation, langage), en cherchant à clarifier leur corrélation constitutive, le lien entre elles.

Mots-clés : Langue, nourriture, sommeil.

\section{INTRODUCTION}

Le sommeil, la nourriture et le langage sont des piliers de la vie saine des enfants, sont étroitement liés dès la naissance et constituent la structure dynamique du développement de l'enfant.

Un tel entrelacement, cependant, n'est pas un postulat unanime, dans les projets de description et de compréhension de cette triade ou chaque partie est prise séparément ou, alors, le privilège d'une partie sur l'autre est souligné, c'est-à-dire qu'il s'agirait de relations qui ne peuvent pas être définies comme implication.

Pour réfléchir à l'implication, il est nécessaire de supposer que dormir, manger et parler impliquent le corps, mais un corps qui exige un nom, un corps subjectif et, par conséquent, un corps enrôlé par le symbolique: dans le lien entre le nom propre et le corps, il y a, dans la lecture de ce trait, quelque chose qui s'articule à travers l'appropriation, de lui-même, qui n'est pas un élément aussi évident et simple dans la constitution humaine (LEITE, 2008, p. 16). Comment une telle appropriation de

RC: 98235

Disponible en: https://www.nucleodoconhecimento.com.br/psychologie-fr/liensymbolique 
l'organisme est-elle représentée, qui vient de son propre organisme lorsqu'il est nommé? Nommer le corps, c'est le reconnaître dans le champ symbolique, effet d'articulations infinies, d'actions et de entrelacer.

Les habitudes de sommeil, d'alimentation et de parole sont des effets de conditions interdépendantes: organiques, psychiques et sociales, qui impliquent l'enfant et résultent, simultanément, d'héritages organiques et symboliques. Ce dernier surdétermine et module l'interaction de l'enfant avec l'environnement, en particulier avec l'autre humain qui s'y trouve.

Pour les bébés, cet environnement peut être représenté, de manière privilégiée, par la figure maternelle, la mère et, il est à noter, ce n'est pas nécessairement la mère biologique ou les soignants, mais une instance mère-assurante, un autre humain qui inscrit le « assistant suivant », le nebensmench freudien, comme l'explique Cabassu (2003). Celui qui, plus que garant de la survie de l'organisme de l'enfant, le reconnaît comme sujet, constitue le lien entre désir (maternel) et parole, à partir duquel la subjectivité et les relations sociales deviennent possibles et s'initie pour le bébé, en nommant son corps et l'inscrire dans un texte d'affiliation et de sociabilité.

$\mathrm{Si}$, comme nous l'avons dit, le sommeil, la nourriture et le langage sont des piliers de la vie de l'enfant, leur implication dépend donc des déclarations maternelles qui lui sont adressées, mais seulement si elles - rassasier la faim, se réchauffer du froid, faire la meute pour dormir... - s'accompagnent d'un désir non anonyme (STORK, LY, MOTA, 1997, p. 34) : c'est la présence de l'autre, l'institution de la relation maternelle.

De plus, il est important de savoir qu'il est nécessaire de reconnaître dans les attitudes maternelles un sens culturel, tout aussi décisif, car les mères interprètent et réagissent aux manifestations [...] du bébé selon les normes de la société à laquelle elles appartiennent, même si leurs réponses sont également modulées par des dynamiques psychiques personnelles (CISMARESCO, 1997, p. 267).

$\mathrm{RC}: 98235$

Disponible en: https://www.nucleodoconhecimento.com.br/psychologie-fr/liensymbolique 
Cette condition présuppose, plus largement, la centralité de la fonction d'une famille de continent, pour laquelle le concept « je-peau » d'Anzieu (1989) peut être éclairant, puisqu'il renvoie aux limites entre le biologique et le symbolique et, pour le bébé, son établissement répond au besoin d'une enveloppe naristique et assure à l'appareil psychique la certitude et la constance d'un bien-être de base (op. cit., p. 44). Ce sentiment de sécurité sera fondamental pour le sentiment d'appartenance, fondamental pour la construction de l'identité de l'enfant.

Cet héritage, à la fois organique et symbolique, transmis dans les relations de l'enfant avec l'autre, allié - bien sûr - à l'héritage organique, dessinera des modèles de conduite et de comportement qui peuvent souvent contribuer à des changements qui compromettent, dans une certaine mesure, le développement général de l'enfant.

Dans la clinique pour enfants, la description des troubles du développement, du plus léger au plus grave, comprend, en règle générale, les aspects liés à la nourriture et au sommeil (WINNICOTT, 1975; MADEIRA, AQUINO, 2003; SANTOS, 2004; JERUSALINSKY, 2004).

Le fait que ces symptômes soient presque toujours alignés les uns avec les autres interroge les cliniciens sur la possibilité qu'il y ait, plus qu'une simple coïncidence, une corrélation entre les fonctions biologiques fondamentales.

De plus, dans les descriptions cliniques des troubles du développement du langage, les rapports de problèmes d'alimentation (PALLADINO, CUNHA, SOUZA, 2007) et de sommeil sont fréquents, ce qui suggère donc une triade de corrélations.

Cependant, et souvent, les différents domaines d'étude du développement ne partagent pas l'idée d'une corrélation significative entre ces fonctions, et il est courant que les questions de sommeil, d'alimentation et de langage soient pensées séparément. Lorsque c'est le cas, en présence de changements de développement, le phénomène est supposé être une comorbidité.

RC: 98235

Disponible en: https://www.nucleodoconhecimento.com.br/psychologie-fr/liensymbolique 
Il y a des exceptions et une telle prédominance, c'est un fait, avec pertinence pour la psychanalyse et certaines approches de la psychologie (GROMANN, 2002), ainsi que pour la petite partie des études de disciplines qui dialoguent avec les deux, comme l'orthophonie, la médecine psychiatrique, la neurologie et l'endocrinologie. Ces exceptions ont suscité une réflexion plus approfondie.

Supposer les corrélations entre les fonctions permet de clarifier l'alignement symptomatologique observé entre les symptômes de l'alimentation, du sommeil et du langage dans la petite enfance. Si tel est le cas, il sera important que le clinicien s'approprie cette perspective, car l'implication déterminera probablement les particularités des procédures de diagnostic et de traitement.

Dans ce sens, il convient d'approfondir et de discuter du développement de ces fonctions (sommeil, alimentation, langage), en cherchant à clarifier leur corrélation constitutive, le lien entre elles.

\section{NOURRITURE ET LANGUE: LA SURPRISE DANS L'OBSERVATION}

II y a quelque temps, dans la vie quotidienne de la clinique pour enfants, une observation du pouvoir des indices a commencé à nous interroger.

Cela s'est produit face à des récits parentaux répétés sur les problèmes d'alimentation dans le cas appelé dans les procédures d'évaluation ou même dans le suivi des plans thérapeutiques dans le cas d'enfants ayant divers problèmes de développement du langage. En traitant avec ces enfants, tôt ou tard, des plaintes au sujet de l'alimentation sont apparues, passant d'idiosyncrasies à des troubles de la déglutition, et leur répétition insistante a été ce qui a gagné en place dans notre écoute clinique et, avec cela, la valeur des indices de l'observation a gagné en pertinence.

RC: 98235

Disponible en: https://www.nucleodoconhecimento.com.br/psychologie-fr/liensymbolique 
Sur la base de ces observations, nous avons structuré la recherche avec un échantillon de 35 patients, et les preuves obtenues ont clarifié une concomitance importante entre le langage et les problèmes alimentaires, suggérant une corrélation significative entre eux (PALLADINO, CUNHA, SOUZA, 2004 et 2007).

Une lecture psychanalytique de la question, proposée par le Revue Française d'Orthophonie (2004), nous a permis d'enrober théoriquement les résultats de cette recherche, nous amenant à réfléchir à la corrélation entre le langage et les problèmes d'alimentation sous la notion d'oralité, telle que proposée par Thibaut (2006, p. 115): la zone orale est l'une des zones égéniques du corps, c'est-à-dire un espace soutenu par un dans lequel de nombreuses fonctions se mélangent dans le plan commun du fonctionnement symbolique. La bouche (organe) est, en ce sens, le territoire de la nourriture, du langage et des affections.

En d'autres termes, la zone orale est le champ somatique dans lequel l'oralité, en tant que plan psychique, entremêle symboliquement la nourriture et le langage.

\section{NOURRITURE, LANGAGE ET SOMMEIL : UNE NOUVELLE ARTICULATION}

Ce repositionnement conceptuel a dirigé et déployé notre écoute clinique vers d'autres espaces, nous amenant, plus récemment, à retravailler la relation entre la nourriture et le langage à partir de l'inclusion du sommeil, formant une triade constitutive de l'enfant. Le sommeil a également été inclus car il est également le protagoniste de la scène fondamentale de la constitution de l'enfant (PALLADINO, 2016, 2018). Le sommeil est lié à la scène de l'alimentation du bébé et fait partie des récits parentaux dans le cas des enfants ayant des problèmes de développement du langage, bien que toujours opaque, c'est-à-dire considérés comme sans valeur ou avec peu de valeur indiciée en termes de risque pour le développement de l'enfant.

$\mathrm{RC}: 98235$

Disponible en: https://www.nucleodoconhecimento.com.br/psychologie-fr/liensymbolique 
En supposant la corrélation constitutive entre ces fonctions, il sera nécessaire de clarifier l'alignement symptomatologique observé entre les symptômes de l'alimentation, du sommeil et du langage, ainsi que d'analyser les implications possibles dans les comportements de diagnostic et de traitement dans ces cas.

Le sommeil, fonction biologique fondamentale, importante pour la restauration du métabolisme énergétique cérébral et la consolidation de la mémoire (CABALLO, NAVARRO, SIERRA, 2002), ainsi que pour l'équilibre psychologique lui-même, résulte d'une organisation temporelle, structurelle et physiologique progressive du sommeil. -rythme d'éveil (GEIB, 2007; PIAULINO DE ARAÚJO, 2012.) C'est un état du cerveau fonctionnant avec deux phases différentes et mesurables : le sommeil REM (Rapid Eye Movement) et le sommeil NREM (No Rapid Eye Movement). Les différences sont principalement en termes de mécanismes métaboliques avec des changements conséquents dans les processus physiologiques et les conditions posturales. Dans le sommeil REM, il y a une augmentation des niveaux métaboliques, une certaine atonie musculaire, une diminution de la température corporelle, un rythme respiratoire équilibré (avec peu et de brèves apnées), des mouvements oculaires rapides et, dans le cas des enfants, des pleurs/sourires/gémissements peuvent se produire. Dans le sommeil NREM, il y a une diminution des niveaux métaboliques, une diminution de la fréquence respiratoire et de la mobilité corporelle (JOHN, 2000). Dans cette phase, il y a un travail intensif de régénération cellulaire et dans l'autre, la phase REM, principalement de régénération psychologique, car c'est là que se déroulent la plupart des rêves.

Le sommeil REM et le sommeil NREM alternent, tout comme le sommeil et l'éveil. Le cycle entre les états de sommeil et d'éveil, initialement déterminé uniquement biologiquement, commence à s'organiser au stade fœtal et sa constitution est étroitement liée au développement du Système Nerveux Central (GEIB, 2007). Progressivement, cette organisation subit l'impact de stimuli exogènes, tels que la

RC: 98235

Disponible en: https://www.nucleodoconhecimento.com.br/psychologie-fr/liensymbolique 
luminosité intense, les sons variés et, surtout, la présence humaine, faisant que le cycle veille-sommeil a un rythme marqué par cette double interférence - endogène et exogène.

À la naissance, le rythme de sommeil du bébé - essentiellement physiologique - est plus simple, avec deux états, actif (sommeil REM) et calme (sommeil NREM), dans un rythme veille-sommeil appelé ultradiane, pas encore dominé par l'alternance journuit. Les cycles alternent dans un temps plus court, et dans les premiers mois, l'état actif est prédominant: le bébé dort à tout moment et se réveille très facilement, et l'éveil est annoncé en pleurant et ce n'est que plus tard que l'éveil silencieux est observé. Au fil du temps, ce rythme est modifié par l'environnement, provoquant un certain atytypage anarchique, c'est-à-dire des changements au début complètement désorganisés et désorganisant le cycle du sommeil, non seulement par les stimuli lumineux et audibles, mais surtout par l'action humaine, qui finit par moduler le rythme qui, finalement, devient cycardien, avec de longues périodes nocturnes de sommeil calme ou même d'éveil plus calme. (PIAULINO DE ARAÚJO 2012; JOHN, 2000)

Une action humaine répandue dans la question du sommeil des bébés est l'alimentation, pour deux raisons principales: elle précède souvent le moment où le bébé dort, outre le fait de compter sur un autre être humain pour son efficacité. Ainsi, ce qui au début n'a une détermination qu'endogène, commence à souffrir de l'influence de l'environnement extérieur. C'est là que les rythmes ultradianiens et cycardiens se consolident et commencent à alterner, le premier pour régir la distribution des phases de sommeil et le second les états de sommeil et d'éveil, dormir la nuit et se réveiller le jour (PIAULINO DE ARAÚJO, 2012; JOHN, 2000).

En prenant le bébé sur ses genoux pour l'allaiter, la mère lui offre un champ affectif inégalé : le plaisir de sisper le lait, d'entendre les mots, d'expérimenter le frottement du corps de l'autre, ce délice le fait s'endormir, se donner.

$\mathrm{RC}: 98235$

Disponible en: https://www.nucleodoconhecimento.com.br/psychologie-fr/liensymbolique 
Le sommeil et la nourriture sont donc au-delà des traces de la nécessité et, plus que cela, constituent un imbrication définitive (PENHA, 2002; FÉDIDA, 1977). Comme mentionné, ces fonctions du bébé dépendent d'un autre être humain pour leur réalisation et, pour cette raison, acquièrent un caractère symbolique, se conformant comme des expériences psychiques. De telles expériences, en général de plaisir, laissent des marques psychiques et des traces cérébrales, créant des souvenirs puissants, qui invitent les sujets à la convivialité avec l'autre.

La mère allaitera son bébé, lui parlera et / ou lui chantera, le caressant, ce qui, avec la satiété, calme l'enfant et favorise le sommeil. Cependant, la faim réveillera, quelque temps plus tard, le bébé, répétant le cycle veille-sommeil.

Cela signifie que ce cycle continu ne sera pas seulement un effet de rythme organique mais aussi d'un rythme psychique, imprimé par la mère (FARIAS, 2004), à travers ses soins.

Autrement dit, le sein (ou le biberon) devient un champ d'échange et un lieu d'insertion du sommeil (FOLINO, LOPES DE SOUZA, 2013), parler/fredonner et bercer constituent l'ethos de ces pratiques de sommeil. Laznik-Penot (1997, p. 37) a montré l'importance cruciale de la mélopeia maternelle - la musique de la voix de la mère parlant au bébé. Cette scène nourricière, à son tour, implique la nourriture, lui donne aussi une existence symbolique.

En fin de compte, ce rituel intime et répété, qui implique le corps et les mots, le sommeil et l'alimentation, constituera la scène fondamentale de l'humanisation, composant le plan relationnel dans lequel le lien entre le bébé et sa mère est inauguré. En fait, cette perspective corrobore les idées de Spinoza (2007) sur les affections et leurs conséquences en termes de joie ou de tristesse : la mère et le bébé affectent et sont affectés l'un par l'autre, ce qui crée un réseau de connexions affectives qui les constituent en tant que tels, élargissant souvent le pouvoir d'agir

$\mathrm{RC}: 98235$

Disponible en: https://www.nucleodoconhecimento.com.br/psychologie-fr/liensymbolique 
dans cette relation singulière et dans toutes les autres. Le pouvoir accru d'agir dans le monde est ce que Spinoza appelle la joie.

Cependant, les affections entre la mère et le bébé ne sont pas toujours augmentées en puissance, parfois le contraire peut se produire. Regardons un exemple relativement courant : les mères qui traversent le baby blues (dépression bénigne, transitoire, potentiellement productive, car elle fait partie des changements générés par la venue d'un bébé) et ne trouvent pas la possibilité d'élaboration et d'acceptation par le contexte social qui ne reconnaît souvent la naissance d'un enfant que par les sentiments de bonheur et de plénitude que, culturellement, il devrait produire (FOLINO, LOPES DE SOUZA, 2013).

Ces mères déprimées passent moins de temps à regarder, à toucher, à parler à leurs bébés, à montrer moins de réactivité, de spontanéité et de taux d'activité plus faibles avec leurs bébés. La dépression post-partum contribue à l'effet de la dyade devenant asynchrone, dans la mesure où la mère est peu ou ne répond pas (SERVILHA, RAAD BUSSAB, 2015). Pendant cette période, le processus affectif entre la mère et le bébé n'augmente pas la puissance des deux, au contraire, il le réduit et peut même dévoiler, dans certains cas, des problèmes ou des troubles psychiques pour les mères et pour le développement du bébé. La diminution du pouvoir d'agir est, à son tour, ce que Spinoza appelle la tristesse.

Il est fondamental que le cycle alimentation-acarcer-sommeil représente des expériences psychiques variées pour le bébé, ce qui implique des sens ambigus pour le bébé: l'alimentation et l'acarération deviennent un espace et un temps d'interaction mère-bébé, et le sommeil de séparation entre eux - lors de l'endormissement, le bébé est placé dans le berceau. Le sommeil représentera une coupure, un intervalle ou une discontinuité dans le lien initial entre l'enfant et sa mère. Le rituel contient, en lui-même, l'ambiguïté entre l'acceptation et la séparation. Cette position de la mère, double, ambiguë, délicate, est mise en scène lorsqu'elle

RC: 98235

Disponible en: https://www.nucleodoconhecimento.com.br/psychologie-fr/liensymbolique 
chante et que l'enfant s'endort pour être placé dans le berceau : approche aimante et séparation.

Peu à peu, la mère espace les coups - une occasion de consolider l'état de veille ainsi que d'introduire des substituts, qui se tiennent entre son propre corps et celui du bébé comme substituts à sa présence: sucettes, casseroles et jouets, même le mot métaphorique, celui qui amène la mère, la représente au bébé. Ces substituts sont insérés pour opérer des déplacements dans la relation fusionnelle qui constitue les premiers jours du lien mère-bébé, sont des supports pour que le bébé fasse face à l'angoisse de la séparation que le sommeil annonce, le sentiment que le sommeil détermine l'absence de la mère, la privation de l'environnement qui assure sa vie et son plaisir.

II convient de noter que la nécessité d'opérer les déplacements pour s'endormir est maintenue et mise à jour à l'âge adulte, dans les rituels qui précèdent le coucher: bains, thés, lectures... . C'est comme si ces rituels supprimaient le « danger » que la solitude du sommeil impose, et garantissaient une sorte de protection d'assurance contre l'engourdissement. Plusieurs personnes âgées manifestent des difficultés à s'endormir par peur de la mort (GEIB, et al, 2003): angoisse de la perte de son monde et du monde. L'adulte a le fonctionnement intériorisé de l'instance d'asseguradora maternelle, opérant les déplacements, créant les substituts de celui qui était le premier gardien du sommeil, maintenant inconsciemment ravivé, par exemple, avec des massages, des pratiques sensuelles, l'ingestion de nourriture, de boissons ou de drogues.

Pendant l'enfance, dans chaque culture et variant en raison des conditions sociales et économiques, il existe plusieurs façons de contourner cette angoisse du sommeil (par la mère et le bébé): co-lit (complet ou partiel), chambre partagée et présence d'allaitement nocturne (nécessaire) (BLAIR, 2008).

$\mathrm{RC}: 98235$

Disponible en: https://www.nucleodoconhecimento.com.br/psychologie-fr/liensymbolique 
Mais, il est important de noter, les cliniciens et les chercheurs ont tendance à se référer à la soi-disant « norme d'or » du sommeil des nourrissons dans les sociétés occidentales soi-disant développées, un modèle recherché tôt: lit simple, chambre séparée des parents, absence d'alimentation nocturne.

Cependant, il existe différentes alertes concernant la question du sommeil, car elle importe également les participants de la scène et les habitudes de sommeil, constitutives externes des cycles de sommeil (GEIB, 2007). Au minimum, on suppose que pour un enfant qui ne s'endort pas ou qui a des réveils successifs, il peut y avoir une mère qui ne pense pas à séparer son corps du corps de son bébé, à arrêter de lui parler, à fredonner pour lui, à le sculpter...

Une possibilité de surmonter cette angoisse est le désir du rêve, un cas dans lequel ce qui a été perdu ou absent peut s'élever oniralement.

Initialement, les études postulaient que les rêves constituaient une activité exclusive de la phase REM du sommeil. Cependant, des recherches plus récentes montrent qu'il n'y a pas d'exclusivité, bien qu'il y ait une prédominance de cette activité dans la phase REM, les chercheurs ayant reconnu qu'elle vise à s'assurer que le sommeil remplit sa fonction régénératrice (GROMANN, 2002).

Les rêves sont un effet de l'engourdissement de la censure, qui empêche les sensations, les sentiments et les actions; une censure qui habite l'esprit de chacun, perturbant à la fois l'éveil et le sommeil lui-même. L'engourdissement fait place à ce qu'on appelle la réalisation de rêves, c'est-à-dire la construction de pensées sans interdiction. Pour cette raison, il a été que les rêves effectuent une restauration psychique, aussi importante que la restauration physique, opérée principalement dans la phase NREM.

Dans le développement de l'enfant, les phases du sommeil sont progressivement suivies pour bien organiser le sommeil, ce qui en fait une toile pour les rêves: du

$\mathrm{RC}: 98235$

Disponible en: https://www.nucleodoconhecimento.com.br/psychologie-fr/liensymbolique 
sommeil physiologique au sommeil symbolique. Le bébé doit construire le chemin entre manger, fermer les yeux, perdre sa mère (hors de vue!) et la retrouver dans des rêves (PENHA, 2002).

En ce qui concerne le sommeil, il existe un étalon-or pour la nourriture, produite principalement culturellement (même si les aspects biologiques y sont considérés). En Occident: allaitement pendant au moins six mois, sevrage naturel, introduction de pâtes et de solides, un chemin qui, rapidement, doit passer d'un liquide chaud, au goût indéfini, à des aliments solides, chauds et salés, dans des scènes familières impliquant des performances individuelles.

Cependant, comme pour la question du sommeil, il y a plusieurs contenus en jeu qui individualisent la scène et méritent l'attention (JERUSALINSKY, 2004; MADEIRA, AQUINO, 2003).

II est possible de faire un parallèle et de dire que pour un enfant qui pleure, s'étouffe, rumine, vomit et/ou refuse de manger, en plus d'éventuels problèmes anatomophysiologiques, il peut y avoir une mère qui a du mal à se positionner comme l'autre assureur du sujet en constitution, effectuant mal la scène alimentaire, sans joie ou même avec tristesse.

Une scène métaphorique de cette difficulté est représentée dans le sevrage, un processus progressif qui mérite la délicatesse, car il est soutenu par des opérations de séparation symboliques et, par conséquent, ce n'est pas un événement « naturel », évident, dont l'approvisionnement en nourriture et le passage de certains types à d'autres sont toujours calmes.

\section{L'ENFILAGE DU LANGAGE}

Le langage est l'action humaine qui prévaut dans les problèmes de sommeil et d'alimentation. Les deux fonctions constituent des expériences symboliques dans les paroles de la mère et dans les paroles enfantines imaginées par la mère. Dans la

$\mathrm{RC}: 98235$

Disponible en: https://www.nucleodoconhecimento.com.br/psychologie-fr/liensymbolique 
chanson, dans l'histoire, dans les interjections de la mère et dans le sucre, dans le regard, dans le gros des yeux, dans les sons inarticulés que sont les «énoncés » du bébé. La scène dialogique est faite comme un « jeu de mots », en interprétation baktinienne. Un dialogue fondateur qui persiste et marque l'entrée du sujet dans la langue, un territoire de rencontres et de confrontations entre subjectivités : choc polyphonique de différentes instances sociales, coexistence conflictuelle de voix et effets de sens. Cela se produit dès le début, à partir de la polyphonie même de la parole maternelle, basée sur une position ambivalente avant votre bébé nouvellement arrivé.

Le discours de la mère ne provoque pas d'effet direct sur son bébé, mais l'atteint par l'effet qu'elle a provoqué en elle-même (CORIAT, 2000), ce qui fait de la relation avec le bébé un champ révélateur, qui peut la lancer dans une expérience de pur plaisir, ou non. Ce qui se concentre immédiatement sur le bébé, c'est la voix de la mère, cette mélodie maternelle, dimension musicale et poétique aux valeurs affectives (LAZNIK-PENOT, 2013), qui implique et soutient le bébé, complètement dépendant. Mélodie qui crée la scène d'alimentation et d'engourdissement. Le bébé porte une attention particulière à la voix de la mère et, en son absence, à toute voix mélodique qui a des affections. C'est ainsi qu'il compose l'interaction avec les autres dans son environnement.

Cette voix-affection, à travers des subtilités, des dynamiques et des rugosités (variation des sens et des sensations), est un espace d'expression spontanée de l'inconscient maternel (FÓNAGY apud LAZNIK-PENOT, 2013). Pour cette raison, il est important de noter si, dans ce discours mélodique, des mots doux sont produits, ou si, à partir de l'ambivalence maternelle, cette voix peut aussi produire, dans ses bruits, d'autres (mots) assez étranges (LAZNIK- PENOT, 2013, p 130). Cela semble être un moyen de comprendre quand l'interaction n'est pas réussie.

$\mathrm{RC}: 98235$

Disponible en: https://www.nucleodoconhecimento.com.br/psychologie-fr/liensymbolique 
Le berceau mélodique du discours de la mère accueille le bébé et le jette dans la dimension symbolique de l'existence humaine, comme le prévient Melgaço (2013, p.10) lorsqu'elle dit que le processus civilisateur met le pouvoir des mots à l'honneur.

À la voix de la mère, le bébé se présente avec son apparence, ses mouvements, ses sons et le va-et-delà entre les deux jeux d'interaction sont assemblés, baignant de mots la relation dans laquelle la mère et le bébé sont situés.

Ce sont des moments constitutifs primordiaux, dans lesquels il est possible d'avoir des impasses et, lorsqu'ils éclosent, des symptômes, des expressions qui trouvent un flux dans la nourriture, le sommeil et le langage, dans la mesure où ces fonctions concernent, centralement, la relation entre le bébé et sa mère.

Dans cette mesure et enfin, le cœur de la question ne réside ni dans la mère ni dans son bébé, mais dans la relation entre eux, dans un processus non pas de similitude, mais d'identification, marqué initialement par le fait que lorsque les bébés ne parlent toujours pas, cette portée relationnelle est anéantie, avant tout, par les processus mentaux de la mère, ce qui serait la première fois de la constitution de la logique psychique et, comme le souligne Vorcaro (2005, p. 24), à partir de l'essaim significatif produit dans le champ de l'Autre, dans lequel l'être vivant est immergé, la «place antérieure » du sujet apparaît comme un effet du langage. Ainsi, ce qui reste inconscient chez la mère est plus sensible aux inscriptions et aux révélations du bébé (CHAVES, 2013, p. 228).

Dormir, manger et, pourquoi pas, regarder/être regardé - les premiers « mots » du bébé - sont le théâtre de révélations pour la maman et son bébé. Dans cette relation singulière, le développement des fonctions piliers dans la vie de l'enfant dépendra du long chemin entre la position d'objet (de et pour la mère) et celle de sujet.

$\mathrm{RC}: 98235$

Disponible en: https://www.nucleodoconhecimento.com.br/psychologie-fr/liensymbolique 


\section{CONSIDÉRATIONS FINALES}

Comme indiqué, cette étude émerge de la clinique et, nous suggérons, qu'elle devrait y revenir. C'est-à-dire que face aux plaintes liées aux troubles du sommeil, de l'alimentation et du langage, il est pertinent que le professionnel de la santé soit en mesure de prendre en charge les demandes possibles résultant de l'articulation symbolique entre ces trois dimensions; articulation inhérente au fonctionnement humain lorsque l'indissociabilité entre le langage, le corps et le psychisme est supposée.

Si c'est le cas, peut-être que le rêve peut également être inclus dans de futures études sur l'implication symbolique complexe travaillée ici.

\section{RÉFÉRENCES}

ANZIEU, D. O Eu-pele. São Paulo: Casa do Psicólogo, 1989.

BLAIR, O. S. O co-leito em perspectiva. Jornal de pediatria, 84(2), 99-101, 2008.

CABALLO, V. E., NAVARRO, J. F. e SIERRA, J. C. Tratamento comportamental dos transtornos do sono In: CARLSON, N. R. Fisiologia do Comportamento. 7ed, SP: Manole, 2002.

Cabassu G. Palavras em torno do berço. In: Wanderley D. B. (org) Palavras em torno do berço. Salvador, Ágalma, 2003.

CHAVES, M. P. C. T. O lugar do analista na clínica com bebês. In: BUSNEL M. C. e MELGAÇO R. G. (orgs) $O$ bebê e as palavras: uma visão transdisciplinar sobre o bebê. Instituto Langage, 2013.

RC: 98235

Disponible en: https://www.nucleodoconhecimento.com.br/psychologie-fr/liensymbolique 
CISMARESCO, A. S. O grito neonatal e suas funções. (seção: o grito e as reações fisiológicas e emocionais das mães). In: BUSNEL, M. C. A linguagem dos bebês. São Paulo, Escuta ed., 1997.

CORIAT, E. Os flamantes bebês e a velha psicanálise. Estilos da Clínica. V.5, n.8, 2000.

FARIAS, C. N. F. e GOMES DE LIMA, G. Relação mãe-criança: esboço de um percurso na teoria psicanalítica. Revista Estilos da Clínica, ano IX, n. 16, 2004.

FÉDIDA, P. Le conte et la zone de l'endormissement. In: Corps de vide et espace de séance. Paris, Jean Pierre Delaye, 1977.

FOLINO, C. S. G. e LOPES DE SOUZA, AS. As Reverberações do encontro mãebebê: sobre a depressão e a depressividade pós-parto. In: BUSNEL, M. C. E MELGAÇO, R. G. (orgs) $O$ bebê e as palavras: uma visão transdisciplinar sobre o bebê. Instituto Langage, 2013.

GEIB, L. T. C., CATALdO NETO,A, WAINBERG R, NUNES ML. Sono e envelhecimento. Rev. Psiquiatria. Rio Gd. Sul, 25(3):453-465, 2003.

GEIB, L. T. C. Desenvolvimento dos estados do sono na infância. Revista Brasileira de Enfermagem, 60(3):323-26, 2007.

GROMANN, R. M. G. Sonho e psiquismo: o labirinto entre o corpo e a subjetividade. Pulsional Revista de psicanálise, 164: 27-34, 2002

JERUSALINSKY, A. Psicanálise e desenvolvimento infantil. Porto alegre, Artes e Ofícios, 2004.

JOHN, M. W. Sensitivity and specificity of the multiple sleep latency test (MSLT), the maintenance of wakefulness test and the Epworth sleepiness scale: failure of the MSLT as a gold standard. J. Sleep Res, 9: 5-11, 2000.

RC: 98235

Disponible en: https://www.nucleodoconhecimento.com.br/psychologie-fr/liensymbolique 
LAZNIK-PENOT, M. C. Rumo à palavra. São Paulo, Escuta ed., 1997.

LAZNIK-PENOT, M. C. Linguagem e comunicação do bebê de zero a três meses. In: LAZNIK-PENOT, M. C. A hora e a vez do bebê. Instituto Langage, 2013.

LEITE, C. A. O. Quando o corpo pede um nome. Tese de doutorado, Instituto de Estudos da Linguagem, UNICAMP, 2008.

MADEIRA, I. R. e AQUINO, L. A. Problemas de abordagem difícil: "não come" e "não dorme". J Pediatr, 79 (Supl 1): 43-54, 2003.

MELGAÇO, R. G. Prefácio. In: BUSNEL, M. C. e MELGAÇO, R. G. (orgs) O bebê e as palavras. São Paulo, Instituto Langage, 2013.

PALLADINO, R. R. R.; CUNHA, M.C. e SOUZA, L. A. P. Transtornos de linguagem e transtornos de alimentação em crianças. Revista Psicanálise e Universidade, 21: 95-108, 2004.

PALLADINO, R. R. R.; CUNHA, M.C. e SOUZA, L. A. P. Transtornos de linguagem e de alimentação: coincidências ou co-ocorrências? Pró-fono Revista de Atualização Científica, 19, 205-214, 2007.

PALLADINO RRR. Linguagem e sono. Anais do XXIV Congresso Brasileiro de Fonoaudiologia, Sociedade Brasileira de Fonoaudiologia, São Paulo, 2016

PALLADINO RRR. Sono e alimentação: funções psíquicas associadas. Anais do III Congresso Iberoamericano de Fonoaudiologia e XXVI Congresso Brasileiro de Fonoaudiologia. Sociedade Brasileira de Fonoaudiologia, Curitiba, 2018.

PENHA, N. C. G. Dormir nos braços da mãe: a primeira guardiã do sono. Rev Psichê, 6(10):65-84, 2002.

$\mathrm{RC}: 98235$

Disponible en: https://www.nucleodoconhecimento.com.br/psychologie-fr/liensymbolique 
PIAULINO DE ARAÚJO, P. D. Validação do questionário do sono infantil de Reimão e Lefèvre (QRL). Tese de doutorado, Departamento de Neurologia, USP, 2012.

RÉÉDUCATION ORTHOPHONIQUE, 44 année, juin/2006, trimestriel n. 226 - La deglutition Dysfunctionnelle, 2006.

SANTOS, M. C. Problemas alimentares da infância sem diagnóstico clínico: quando vigiar, quando atuar? Rev. Hospital de Crianças Maria Pia, vol. XIII, n.4: 342-7, 2004.

SERVILHA, B. e RAAD BUSSAB, V.S. Interação Mãe-Criança e Desenvolvimento da Linguagem: a Influência da Depressão Pós-Parto. Psico, Porto Alegre, v. 46, n. 1, pp. 101-109, jan.-mar. 2015.

SPINOZA, B. Ética. Trad. e notas de Thomaz Tadeu. Belo Horizonte, Autêntica Ed. 2007.

STORK, H.; LY, O. e MOTA. G. Os bebês falam: como você os compreende? Uma comparação intercultural. In: BUSNEL, M. C. (org) A linguagem dos bebês. Sabemos escutá-los? São Paulo: Escuta Ed., 1997.

THIBAULT, C. A língua, órgão chave das oralidades. Rééducation Orthophonique, 44 année, juin/2006, trimestriel, n.226 - La deglutition Dysfunctionnelle, p.115, 2006.

VORCARO, A. Crianças em Psicanálise, Rio de Janeiro, Companhia de Freud, 2005.

WINNICOTT, D. W. Pensando sobre crianças. Porto Alegre, Artmed, 1975.

Soumis: Septembre, 2021.

Approbation : Septembre, 2021.

RC: 98235

Disponible en: https://www.nucleodoconhecimento.com.br/psychologie-fr/liensymbolique 